\title{
Blow-up properties for a degenerate parabolic system coupled via nonlinear boundary flux
}

Si $X u^{*}$

\section{"Correspondence:}

xusi_math@hotmail.com Department of Mathematics, Jiangxi Vocational College of Finance And Economics, Jiujiang, Jiangxi 332000, P.R. China

\begin{abstract}
In this paper, we study the simultaneous and non-simultaneous blow-up problem for a system of two nonlinear diffusion equations in a bounded interval, coupled at the boundary in a nonlinear way. Under certain hypotheses on the initial data and parameters, we prove that non-simultaneous blow-up is possible. Moreover, we get some conditions on which simultaneous blow-up must occur, as well as the non-simultaneous blow-up conditions for every initial data. Furthermore, we get a result on the coexistence of both simultaneous and non-simultaneous blow-ups.
\end{abstract}

MSC: 5B33; 35K65; 35K55

Keywords: non-simultaneous blow-up; blow-up rate; nonlinear diffusion; nonlinear boundary flux

\section{Introduction and main results}

In this paper, we study the nonlinear parabolic system

$$
\left\{\begin{array}{l}
u_{t}=\left(u^{m}\right)_{x x}+\lambda_{1} u^{\alpha}, \\
v_{t}=\left(v^{n}\right)_{x x}+\lambda_{2} v^{\beta},
\end{array} \quad(x, t) \in D_{T}=(0,1) \times(0, T),\right.
$$

with nonlinear coupling boundary conditions

$$
\left\{\begin{array}{l}
\left(u^{m}\right)_{x}(1, t)=u^{p_{1}} v^{q_{1}}(1, t), \\
\left(v^{n}\right)_{x}(1, t)=u^{p_{2}} v^{q_{2}}(1, t), \quad t \in(0, T), \\
\left(u^{m}\right)_{x}(0, t)=0, \\
\left(v^{n}\right)_{x}(0, t)=0
\end{array}\right.
$$

and the initial data

$$
\left\{\begin{array}{l}
u(x, 0)=u_{0}(x), \\
v(x, 0)=v_{0}(x),
\end{array} \quad x \in[0,1],\right.
$$

where $m, n>1, \alpha, \beta, p_{1}, q_{2} \geq 0, p_{2}, q_{1}>0$, the initial conditions $u_{0}, v_{0} \geq \delta>0$, are continuous bounded and satisfy 


$$
\left\{\begin{array}{l}
u_{0}^{\prime} \geq 0, \quad\left(u_{0}^{m}\right)^{\prime \prime}+\lambda_{1} u_{0}^{\alpha} \geq 0, \\
v_{0}^{\prime} \geq 0, \quad\left(v_{0}^{n}\right)^{\prime \prime}+\lambda_{2} v_{0}^{\beta} \geq 0,
\end{array} \quad x \in[0,1] .\right.
$$

Remark 1.1 Combining (1.4) with the comparison principle, we can actually get following relationships:

$$
u_{t}, u_{x}, v_{t}, v_{x} \geq 0, \quad u, v \geq \delta>0, \quad \text { for all } x \in[0,1] \text { and } t \in[0, T) .
$$

The reaction-diffusion system (1.1)-(1.3) can be used to describe heat transfer in a mixed medium with absorptions and nonlinear boundary flux, and some chemical reaction processes with the slow diffusion phenomenon (see $[1,2])$.

In [3], Song and Zheng considered the blow-up conditions of the following problem:

$$
\begin{cases}\left(u^{m}\right)_{t}=\Delta u+u^{\alpha_{1}} v^{p_{1}}, \quad\left(v^{n}\right)_{t}=\Delta v+u^{q_{1}} v^{\beta_{1}}, & (x, t) \in \Omega \times(0, T), \\ \frac{\partial u}{\partial \eta}=u^{\alpha_{2}} v^{p_{2}}, \quad \frac{\partial v}{\partial \eta}=u^{q_{2}} v^{\beta_{2}}, & (x, t) \in \partial \Omega \times(0, T), \\ u(x, 0)=u_{0}(x), \quad v(x, 0)=v_{0}(x), & x \in \bar{\Omega},\end{cases}
$$

where $\Omega$ is a bounded domain in $R^{N}, m, n>0, \alpha_{i}, \beta_{i}, p_{i}, q_{i} \geq 0$. If we let $u^{m}=\bar{u}, v^{n}=\bar{v}$, $N=1, p_{1}=q_{1}=0$, we just get system (1.1)-(1.3). From the conclusions in [3], we know that the solution of system (1.1)-(1.3) blows up if and only if one of the following conditions holds:

(1) $\max \left\{\alpha, p_{1}\right\}>1$,

(2) $\max \left\{\beta, q_{2}\right\}>1$,

(3) $p_{2} q_{1}>\left(1-p_{1}\right)\left(1-q_{2}\right)$.

In this case we can only have

$$
\lim _{t \rightarrow T} \sup \left\{\|u(\cdot, t)\|_{\infty}+\|v(\cdot, t)\|_{\infty}\right\}=\infty
$$

However, from the above result, we cannot show that blow-up is simultaneous or nonsimultaneous. The blow-up rate is not known yet.

Recently, the simultaneous and non-simultaneous blow-up problems of parabolic systems have been widely considered by many authors [4-14]. For example, when $m=n=1$, $\lambda_{i}=0$, Pinasco and Rossi [6] considered the system of heat equations coupled via a nonlinear boundary flux with $\Omega \subset R^{N}$ and found that $u$ blows up at time $T$ and $v$ remains bounded up to time $T$ for certain initial data if and only if $p_{1}>1$ and $p_{2}<p_{1}-1$.

When $\lambda_{i}=0, \alpha=\beta=0, m>0, n>0$, Brändle et al. [4] studied problem (1.1)-(1.3) and proved that the non-simultaneous blow-ups occur if and only if

$$
2 p_{2}<\max \left\{p_{1}-1,2 p_{1}-(m+1)\right\} .
$$

They also found some conditions under which $u$ blows up and $v$ remains bounded for every initial data.

When $m=n=1$ and $\lambda_{i}<0$, Zheng and Qiao [10] also got the sufficient and necessary conditions of non-simultaneous blow-up.

In this paper, by using a modification of methods in [4] and [10] we will focus on the simultaneous and non-simultaneous blow-up problems to (1.1)-(1.3), and we get our main results as follows. 
Theorem 1.1 When $\lambda_{i}>0$, if $\max \left\{\alpha, p_{1}\right\}>m$ and

$$
\max \left\{2 p_{1}-m, \alpha\right\}>2 p_{2}+1,
$$

then there exists initial data $\left(u_{0}, v_{0}\right)$ such that $u$ blows up at a finite time $T$, while $v$ remains bounded up to $T$.

Theorem 1.2 When $\lambda_{i}>0$ and $\max \left\{\alpha, p_{1}\right\}>m$, if $u$ blows up at time $T$ and $v$ remains bounded up to $T$, then (1.5) holds.

Theorem 1.3 When $\lambda_{i}<0$, if $p_{1}>m$ and $2 p_{1}-m>\max \left\{2 p_{2}+1, \alpha\right\}$, then there exists initial data $\left(u_{0}, v_{0}\right)$ such that $u$ blows up at a finite time $T$, while $v$ remains bounded up to $T$.

Remark 1.2 When $m=n=1$ and $\lambda_{i}<0$, Theorem 1.3 is consistent with Theorem 1 in [10].

By interchanging the roles of $u$ and $v$, we get the following results.

Corollary 1.4 When $\lambda_{i}>0$,

(i) if $\max \left\{\beta, q_{2}\right\}>n$ and

$$
\max \left\{2 q_{2}-n, \beta\right\}>2 q_{1}+1
$$

then there exists initial data $\left(u_{0}, v_{0}\right)$ such that $v$ blows up at a finite time $T$, while $u$ remains bounded up to $T$.

(ii) When $\max \left\{\beta, q_{2}\right\}>n$, if $v$ blows up at a finite time $T$ and $u$ remains bounded up to $T$, then (1.6) holds.

Corollary 1.5 When $\lambda_{i}<0$, if $q_{2}>n$ and $2 q_{2}-n>\max \left\{2 q_{1}+1, \beta\right\}$, then there exists initial data $\left(u_{0}, v_{0}\right)$ such that $v$ blows up at a finite time $T$, while $u$ remains bounded up to $T$.

Theorem 1.6 When $\lambda_{i}>0$, if $\max \left\{\alpha, p_{1}\right\}>m$ and $2 p_{2}+1 \geq \max \left\{2 p_{1}-m, \alpha\right\}$, then for any initial data $\left(u_{0}, v_{0}\right)$ the solution $(u, v)$ to (1.1)-(1.3) blows up simultaneously at a finite time $T$.

Theorem 1.7 When $\lambda_{i}>0$, if $\max \left\{\alpha, p_{1}\right\}>m$ and (1.5) holds, then the set of initial data such that $u$ blows up and $v$ remains bounded is open in the $L^{\infty}$ topology. If $\max \left\{\beta, q_{2}\right\}>n$ and (1.6) holds, then the set of initial data such that $v$ blows up and $u$ remains bounded is open in $L^{\infty}$ topology.

Next, inspired by $[9,12]$, we consider the coexistence of both simultaneous and nonsimultaneous blow-ups, and we get the blow-up rate if simultaneous blow-ups occur.

Theorem 1.8 When $\lambda_{i}>0$, if

$$
\left\{\begin{array}{l}
\max \left\{\alpha, p_{1}\right\}>m, \\
2 p_{1}-m>\max \left\{\alpha, 2 p_{2}+1\right\},
\end{array}\right.
$$


and

$$
\left\{\begin{array}{l}
\max \left\{\beta, q_{2}\right\}>n, \\
2 q_{2}-n>\max \left\{\beta, 2 q_{1}+1\right\},
\end{array}\right.
$$

then there may occur both simultaneous and non-simultaneous blow-ups. Moreover, if $(u, v)$ blow up simultaneously, then there exist positive constants $c, C$ such that

$$
\begin{aligned}
& c(T-t)^{-\theta_{1}} \leq \max _{[0,1]} u(\cdot, t) \leq C(T-t)^{-\theta_{1}}, \\
& c(T-t)^{-\theta_{2}} \leq \max _{[0,1]} v(\cdot, t) \leq C(T-t)^{-\theta_{2}}, \quad \text { as } t \rightarrow T,
\end{aligned}
$$

where

$$
\begin{aligned}
& \theta_{1}=\frac{n-2 q_{2}+2 q_{1}+1}{4 p_{2} q_{1}-\left(n-2 q_{2}+1\right)\left(m-2 p_{1}+1\right)}, \\
& \theta_{2}=\frac{m-2 p_{1}+2 p_{2}+1}{4 p_{2} q_{1}-\left(n-2 q_{2}+1\right)\left(m-2 p_{1}+1\right)} .
\end{aligned}
$$

Finally, let us show that, under certain conditions, blow-up is always non-simultaneous.

Theorem 1.9 When $\lambda_{i}>0$, if

$$
\left\{\begin{array}{l}
p_{1}>\max \left\{m, \frac{m+\alpha}{2}\right\} \\
q_{2}>\max \left\{n, \frac{n+\beta}{2}\right\}
\end{array}\right.
$$

and

$$
\left\{\begin{array}{l}
n-2 q_{2}+2 q_{1}+1 \geq 0 \\
m-2 p_{1}+2 p_{2}+1<0
\end{array}\right.
$$

then $u$ blows up and $v$ remains bounded for any initial data.

Theorem 1.10 When $\lambda_{i}>0$, if $\max \left\{\alpha, p_{1}\right\}>m, \max \left\{\beta, q_{2}\right\} \leq 1$ and (1.5) holds, then $u$ blows up and $v$ remains bounded for any initial data.

The rest of this paper is organized as follows. In the next section, we first get some blowup results of scalar problems, and using them to prove Theorems 1.1-1.3. In Section 3, we consider the coexistence of both simultaneous and non-simultaneous blow-ups, Theorems 1.7, 1.8 are proved. In Section 4, we show that non-simultaneous blow-ups always occur, and we prove Theorems 1.9 and 1.10 .

\section{Non-simultaneous and simultaneous blow-up}

In this section, in order to prove Theorems 1.1-1.3, we will start with the following problem:

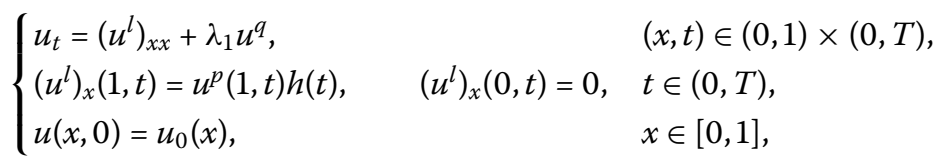


where $l>1, q, p>0$, and $\lambda_{1} \in R, h(t)$ is a continuous, bounded, nondecreasing, and strictly positive function.

When $h=1, \lambda_{1}=1$, problem (2.1) has been already studied in [15-17]. There, it is shown that solutions blow up if and only if

$$
\max \{q, p\}>1
$$

By the comparison principle, when $\lambda_{1}>0$, the solutions of (2.1) blow up if and only if the same restriction.

When $\lambda_{1}<0$, we know from [18], if $p>l$ and $q<2 p-l$, that the solutions of (2.1) blow up for large initial data.

We give the blow-up rate of the solution of (2.1) in the following two lemmas.

Lemma 2.1 Let $u$ be a solution of (2.1) with $\lambda_{1}>0$, if $\max \{q, p\}>l$, then there exist positive constants $c$ and $C$, such that

$$
c(T-t)^{-\sigma} \leq \max _{[0,1]} u(\cdot, t) \leq C(T-t)^{-\sigma} .
$$

(i) When $2 p>q+l, \sigma=\frac{1}{2 p-l-1}$.

(ii) When $2 p<q+l, \sigma=\frac{1}{q-1}$.

(iii) When $2 p=q+l, \sigma=\frac{1}{q-1}=\frac{1}{2 p-l-1}$.

Proof Notice that if case (i) holds, by $\max \{q, p\}>l$ and $2 p>q+l$, we get $p>l$. If case (ii) holds, it follows from $\max \{q, p\}>l$ and $2 p<q+l$ that $q>l$. Thus, by a similar proof to that of Theorem 3.1 in [15], we can prove easily Lemma 2.1. We omit it here.

Lemma 2.2 When $\lambda_{1}<0, p>l$, and $2 p>q+l$. If $u$ be a solution of (2.1), then there exist positive constants $c$ and $C$, such that

$$
c(T-t)^{-\sigma} \leq \max _{[0,1]} u(\cdot, t) \leq C(T-t)^{-\sigma},
$$

where $\sigma=\frac{1}{2 p-l-1}$.

Proof The proof is similar to that of Theorem 1.2 in [18]. We omit it here.

Furthermore, we need to consider the following problem.

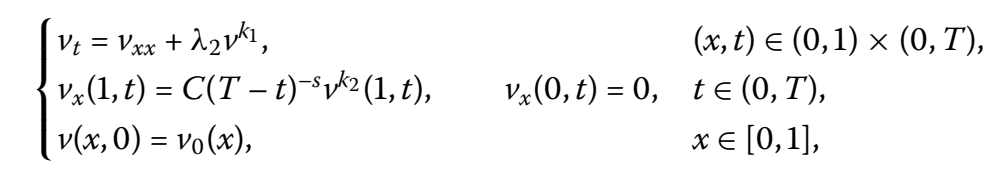

where $k_{i}, s>0$ and $\lambda_{2} \in R$.

Lemma 2.3 If $s<1 / 2$, then given $v_{0}$ there exists $T$ small enough such that the solution of (2.4) verifies

$$
\sup _{0<t<T}\|v(\cdot, t)\|_{\infty}<2\left\|v_{0}\right\|_{\infty} .
$$


Proof For given $v_{0}$, we denote

$$
K=\max _{[0,1]} v_{0}=v_{0}(1)
$$

let $t_{0}$ be the first time that

$$
\max _{[0,1]} v\left(\cdot, t_{0}\right)=v\left(1, t_{0}\right)=2 K
$$

If such $t_{0}$ does not exist, $v$ will remain bounded with $v<2 K$, the conclusion follows.

Let $\Gamma(x, t)$ be the fundamental solution of the heat equation in $[0,1]$, so

$$
\Gamma(x, t)=\frac{1}{2 \sqrt{\pi t}} \exp \left\{\frac{-x^{2}}{4 t}\right\} .
$$

It is well known that $\Gamma$ satisfies (see [19]),

$$
\begin{aligned}
& \int_{0}^{1} \Gamma(x-y, t-z) d y \leq 1, \\
& \int_{z}^{t} \Gamma(1, t-\tau) \frac{1}{2(t-\tau)} d \tau \leq C^{*} \sqrt{t-z}, \\
& \int_{z}^{t} \Gamma(0, t-\tau) d \tau=\frac{1}{\sqrt{\pi}} \sqrt{t-z}, \\
& \frac{\partial \Gamma}{\partial \eta_{y}}(x-y, t-\tau)=\frac{x-y}{2(t-\tau)} \Gamma(x-y, t-\tau), \quad x, y \in[0,1], 0 \leq z<t .
\end{aligned}
$$

By Green's identity of (2.4), we have

$$
\begin{aligned}
v(x, t)= & \int_{0}^{1} \Gamma(x-y, t-\tau) \nu(y, z) d y+\int_{z}^{t} \int_{0}^{1} \Gamma(x-y, t-\tau)\left(\lambda_{2} v^{k_{1}}(y, \tau)\right) d y d \tau \\
& +\int_{z}^{t} \frac{\partial v}{\partial x}(1, \tau) \Gamma(x-1, t-\tau) d \tau-\int_{z}^{t} \frac{\partial \Gamma}{\partial \eta_{y}}(x-1, t-\tau) \nu(1, \tau) d \tau \\
& +\int_{z}^{t} \frac{\partial \Gamma}{\partial \eta_{y}}(x, t-\tau) \nu(0, \tau) d \tau,
\end{aligned}
$$

where $0 \leq z<t<T, 0<x<1$. With $z=0$, and $x \rightarrow 1$, it follows that

$$
\begin{aligned}
v(1, t) \leq & v_{0}(1) \int_{0}^{1} \Gamma(1-y, t) d y+\int_{0}^{t} \int_{0}^{1} \Gamma(1-y, t-\tau) d y \lambda_{2} v^{k_{1}}(1, \tau) d \tau \\
& +\int_{0}^{t} C(t-\tau)^{-s} v^{k_{2}}(1, \tau) \frac{1}{2 \sqrt{\pi}}(t-\tau)^{-1 / 2} d \tau+v(1, t) \int_{0}^{t} \frac{1}{2(t-\tau)} \Gamma(1, t-\tau) d \tau .
\end{aligned}
$$

Furthermore, we have

$$
\begin{aligned}
v\left(1, t_{0}\right) \leq & v_{0}(1)+\lambda_{2} v^{k_{1}}\left(1, t_{0}\right) \\
& +\frac{C}{2 \sqrt{\pi}} v^{k_{2}}\left(1, t_{0}\right) \int_{0}^{t_{0}}\left(t_{0}-\tau\right)^{-s-1 / 2} d \tau+v\left(1, t_{0}\right) C^{*} \sqrt{t_{0}} .
\end{aligned}
$$


(i) If $\lambda_{2}<0,(2.5)$ becomes

$$
v\left(1, t_{0}\right) \leq v_{0}(1)+\frac{C}{2 \sqrt{\pi}} v^{k_{2}}\left(1, t_{0}\right) \int_{0}^{t_{0}}\left(t_{0}-\tau\right)^{-s-1 / 2} d \tau+v\left(1, t_{0}\right) C^{*} \sqrt{t_{0}} .
$$

Since $s<1 / 2$, for $N=2^{k_{2}} K^{k_{2}-1}+3$, if we choose $T$ sufficiently small enough, $\int_{0}^{t_{0}}\left(t_{0}-\right.$ $\tau)^{-s-1 / 2} d \tau$ can be smaller than $2 \sqrt{\pi} / N C$, also $\sqrt{t_{0}} \leq \sqrt{T} \leq 1 /\left(N C^{*}\right)$. Thus

$$
\frac{N-1}{N} v\left(1, t_{0}\right) \leq v_{0}(1)+\frac{1}{N} v^{k_{2}}
$$

since $v\left(1, t_{0}\right)=2 K$,

$$
\frac{2(N-1)}{N} K \leq K+\frac{1}{N}(2 K)^{k_{2}},
$$

and hence

$$
N \leq 2^{k_{2}} K^{k_{2}-1}+2
$$

this is a contradiction.

(ii) If $\lambda_{2}>0$, for

$$
N=\frac{(2 K)^{k_{2}}+2 K}{K+\lambda_{2}(2 K)^{k_{1}}}+1
$$

if we choose $T$ sufficiently small enough, $\int_{0}^{t_{0}}\left(t_{0}-\tau\right)^{-s-1 / 2} d \tau$ can be smaller than $2 \sqrt{\pi} / N C$, also $\sqrt{t_{0}} \leq \sqrt{T} \leq 1 /\left(N C^{*}\right)$. Thus

$$
v\left(1, t_{0}\right) \leq v_{0}(1)+\frac{1}{N} v^{k_{2}}\left(1, t_{0}\right)+\lambda_{2} v^{k_{1}}\left(1, t_{0}\right)+\frac{1}{N} v\left(1, t_{0}\right)
$$

and hence

$$
N \leq \frac{(2 K)^{k_{2}}+2 K}{K+\lambda_{2}(2 K)^{k_{1}}}
$$

this is also a contradiction.

Lemma 2.4 For given $v_{0}$, let $v(x, t)$ be the solution of the following problem:

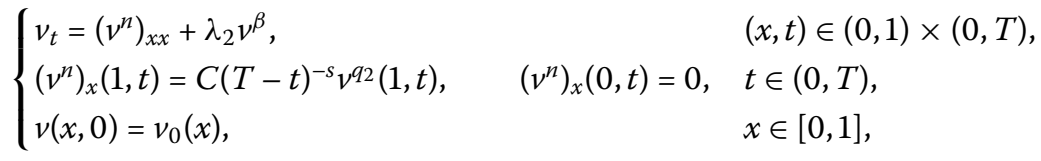

where $n>1, \beta, q_{2}, s>0$, and $\lambda_{2} \in R$. If $s<1 / 2$, then there exists $T$ small enough such that $v(x, t)$ satisfies

$$
\sup _{0<t<T}\|v(\cdot, t)\|_{\infty}<2\left\|v_{0}\right\|_{\infty}
$$


Proof Given $v_{0}$, let $z$ be a solution of (2.4), with $z_{0}(x)=v_{0}^{n}(x), k_{1}=\frac{\beta}{n}, k_{2}=\frac{q_{2}}{n}$. A constant $C\left(z_{0}\right)=\left(n\left(2\left\|z_{0}\right\|_{\infty}\right)^{\frac{n-1}{n}}\right)^{-1}$ in front of $z_{t}$. Let $\bar{v}=z^{1 / n}$, then

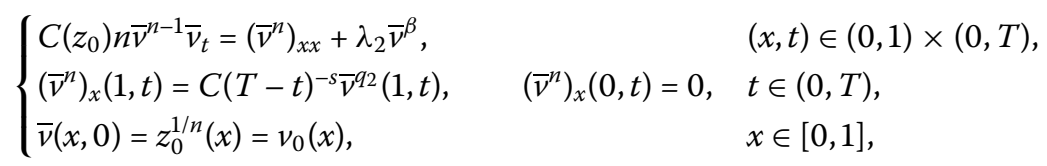

since $s<1 / 2$, from Lemma 2.3, we know that a small enough $T$ can be found, such that

$$
\sup _{0<t<T}\|z(\cdot, t)\|_{\infty}<2\left\|z_{0}\right\|_{\infty}
$$

$z$ is also bounded away from zero and $z_{t}>0$, so we have

$$
n \bar{\nu}^{n-1}<\frac{1}{C\left(z_{0}\right)}
$$

Therefore $\bar{v}$ is a supersolution of problem (2.7). Hence

$$
\sup _{0<t<T}\|v(\cdot, t)\|_{\infty} \leq \sup _{0<t<T}\|\bar{v}(\cdot, t)\|_{\infty}<\left(2\left\|v_{0}\right\|_{\infty}^{n}\right)^{\frac{1}{n}}
$$

Lemma 2.5 If $n>1$ and $s \geq 1 / 2$, then every nonnegative, nontrivial solution of the following problem blows up at time $T$,

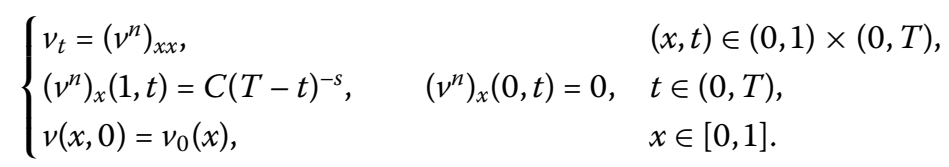

Proof It is an immediate conclusion of Theorem 3.2(i) in [4].

Proof of Theorem 1.1 First, we consider $\underline{u}$ to be a solution of the following problem:

$$
\left\{\begin{array}{lll}
\underline{u}_{t}=\left(\underline{u}^{m}\right)_{x x}+\lambda_{1} \underline{u}^{\alpha}, & (x, t) \in(0,1) \times\left(0, T^{\prime}\right), \\
\left(\underline{u}^{m}\right)_{x}(1, t)=\delta^{q_{1}} \underline{u}^{p_{1}}(1, t), & \left(\underline{u}^{m}\right)_{x}(0, t)=0, & t \in\left(0, T^{\prime}\right), \\
\underline{u}(x, 0)=u_{0}(x), & x \in[0,1]
\end{array}\right.
$$

Since $\max \left\{\alpha, p_{1}\right\}>m>1, \underline{u}$ blows up at finite time $T^{\prime}$. From Lemma 2.1, we also have the estimate

$$
\underline{u}(\cdot, t) \leq C\left(T^{\prime}-t\right)^{-\sigma}, \quad \text { for all }(x, t) \in[0,1] \times\left[0, T^{\prime}\right) .
$$

In particular, $u_{0}(x)=u(x, 0) \leq C\left(T^{\prime}\right)^{-\sigma}$, that is, $T^{\prime} \leq\left(\frac{C}{u_{0}(x)}\right)^{1 / \sigma}$.

Since $v \geq \delta>0$, for all $(x, t) \in[0,1] \times[0, T), u$ is a supersolution of $\underline{u}$. Then $u$ also blows up, and the blow-up time $T$ is smaller than $T^{\prime}$. Thus we have $T \leq T^{\prime} \leq\left(\frac{C}{u_{0}(x)}\right)^{1 / \sigma}$. As $\sigma>0$, we can choose the initial data $u_{0}(x)$ large enough such that $T$ is small.

Given $v_{0}$, we denote

$$
K=\max _{[0,1]} v_{0}=v_{0}(1) .
$$


We claim that there exists $u_{0}$ large enough such that $v<3 K$ for all $(x, t) \in[0,1] \times[0, T]$. If this claim is not true, there exists $0<t_{0}<T$, which is the first time $v\left(1, t_{0}\right)=3 K$. So when $t \in\left[0, t_{0}\right], v \leq 3 K$, when $t \in\left[t_{0}, T\right], v \geq 3 K$. We denote a cutoff function

$$
\widetilde{v}(x, t)= \begin{cases}v(x, t), & (x, t) \in[0,1] \times\left[0, t_{0}\right], \\ 3 K, & (x, t) \in[0,1] \times\left[t_{0}, T\right]\end{cases}
$$

obviously, $\widetilde{v}$ is a bounded, continuous, and nondecreasing function.

Let $\tilde{u}$ be a solution of following problem:

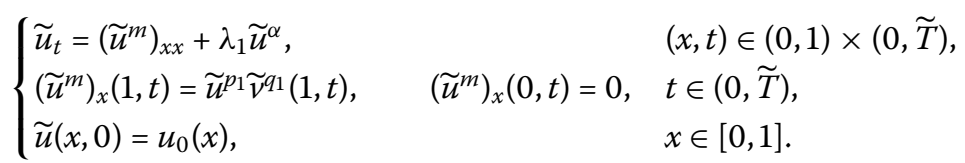

$\widetilde{u}$ blows up at $\widetilde{T}$, by Lemma 2.1 ,

$$
\widetilde{u}(\cdot, t) \leq C(\widetilde{T}-t)^{-\sigma}, \quad \text { for all }(x, t) \in[0,1] \times[0, \widetilde{T}) .
$$

As $\widetilde{v} \leq v$ for $(x, t) \in[0,1] \times[0, T], \widetilde{u}$ is a subsolution of $u$, thus we have $T \leq \widetilde{T}$. Therefore,

$$
u(1, t)=\widetilde{u}(1, t) \leq C(\widetilde{T}-t)^{-\sigma} \leq C(T-t)^{-\sigma}, \quad 0<t \leq t_{0} .
$$

Now we consider the equations of $v$,

$$
\begin{cases}v_{t}=\left(v^{n}\right)_{x x}+\lambda_{2} v^{\beta}, & (x, t) \in(0,1) \times\left(0, t_{0}\right), \\ \left(v^{n}\right)_{x}(1, t)=u^{p_{2}} v^{q_{2}}(1, t) \leq C(T-t)^{-p_{2} \sigma} v^{q_{2}}(1, t), & t \in\left(0, t_{0}\right), \\ \left(v^{n}\right)_{x}(0, t)=0, & t \in\left(0, t_{0}\right), \\ v(x, 0)=v_{0}(x), & x \in[0,1] .\end{cases}
$$

So $v$ is a subsolution of (2.7). Let $\bar{v}$ be the solution of (2.7), where $s=p_{2} \sigma$.

(i) If $2 p_{1}>\alpha+m$, from (1.5) we have $2 p_{1}-m>2 p_{2}+1$, thus $s=p_{2} \sigma=\frac{p_{2}}{2 p_{1}-m-1}<1 / 2$.

(ii) If $2 p_{1}<\alpha+m$, from (1.5) we have $\alpha>2 p_{2}+1$, also get $s=\frac{p_{2}}{\alpha-1}<1 / 2$.

(iii) If $2 p_{1}=\alpha+m$, from (1.5) we have $2 p_{1}-m=\alpha=2 p_{2}+1$, and we get

$$
s=\frac{p_{2}}{\alpha-1}=\frac{p_{2}}{2 p_{1}-m-1}<1 / 2
$$

By Lemma 2.4, we have $\bar{v}\left(1, t_{0}\right)<2 K$. But on the other hand, we have

$$
3 K=v\left(1, t_{0}\right) \leq \bar{v}\left(1, t_{0}\right)<2 K,
$$

which is a contradiction; we conclude that $v$ remains bounded up to time $T$.

Proof of Theorem 1.2 Let $h(t)=v(1, t)$. Since $\max \left\{\alpha, p_{1}\right\}>m$, and $v$ is a bounded, continuous nondecreasing function in $[0, T]$, by Lemma 2.1 , we know

$$
c(T-t)^{-\sigma} \leq \max _{[0,1]} u(\cdot, t) .
$$


Since $v \geq \delta, v$ satisfies

$$
\left\{\begin{array}{l}
v_{t}=\left(v^{n}\right)_{x x}+\lambda_{2} v^{\beta} \geq\left(v^{n}\right)_{x x} \\
\left(v^{n}\right)_{x}(1, t)=u^{p_{2}} v^{q_{2}}(1, t) \geq c \delta^{q_{1}}(T-t)^{-p_{2} \sigma} \\
\left(v^{n}\right)_{x}(0, t)=0 \\
v(x, 0)=v_{0}(x)
\end{array}\right.
$$

So $v$ is a supersolution of (2.8). Let $\underline{v}$ be the solution of (2.8) with $s=p_{2} \sigma$. By Lemma 2.5, we have $s<1 / 2$, otherwise $\underline{v}$ will blow up, and $v$ cannot be bounded.

(i) If $2 p_{1}>\alpha+m$, from $s=\frac{p_{2}}{2 p_{1}-m-1}<1 / 2$, we have $2 p_{1}-m>\max \left\{2 p_{2}+1, \alpha\right\}$.

(ii) If $2 p_{1}<\alpha+m$, from $s=\frac{p_{2}}{\alpha-1}<1 / 2$, we have $\alpha>\max \left\{2 p_{2}+1,2 p_{1}-m\right\}$.

(iii) If $2 p_{1}=\alpha+m$, from $s=\frac{p_{2}}{\alpha-1}=\frac{p_{2}}{2 p_{1}-m-1}<1 / 2$, we have $2 p_{1}-m=\alpha>2 p_{2}+1$.

From the previous three cases, we have (1.5).

Proof of Theorem 1.3 By using the blow-up rate estimate in Lemma 2.2 instead of the estimate in Lemma 2.1, Theorem 1.3 can be proved by the same steps as in the proof of statement (i) in Theorem 1.1. We omit it here.

Proof of Theorem 1.6 Under our assumptions $u$ must blow up. If there exists initial data that $u$ blows up at finite time $T$, while $v$ remain bounded up to that time, from Theorem 1.2 we have (1.5) holds, which is a contradiction.

\section{Coexistence of simultaneous and non-simultaneous blow-ups}

In this section, we consider the coexistence of both simultaneous and non-simultaneous blow-ups, and we prove Theorems 1.7 and 1.8.

Proof of Theorem 1.7 Let $(u, v)$ be a solution of (1.1)-(1.3) with initial data $\left(u_{0}, v_{0}\right)$ such that $u$ blows up at $T$ while $v$ remains bounded, that is, $v \leq C$. We only need to find a $L^{\infty}$ neighborhood of $\left(u_{0}, v_{0}\right)$ such that any solution $(\hat{u}, \hat{v})$ of $(1.1)-(1.3)$ with initial data $\left(\hat{u_{0}}, \hat{v_{0}}\right)$ in this neighborhood maintains the property that $u$ blows up while $v$ remains bounded.

In fact, as the solutions of (1.1)-(1.3) are bounded up to time $T-\varepsilon$, the continuity of solution respect to initial conditions holds up to $T-\varepsilon$, which means for any $\epsilon_{0}$, there is a $\delta_{0},\left\|\left(\hat{u_{0}}, \hat{v_{0}}\right)-\left(u_{0}, v_{0}\right)\right\|_{\infty}<\delta_{0}$, such that $\|(\hat{u}, \hat{v})-(u, v)\|_{\infty}<\epsilon_{0}$. When we let $\epsilon_{0}=1$, there is a $\delta_{0}$, such that $\|(\hat{u}, \hat{v})-(u, v)\|_{\infty}<1$, so we get $\|\hat{u}-u\|_{\infty}<1$ and $\|\hat{v}-v\|_{\infty}<1$. Moreover, $u$ becomes large at time $T-\varepsilon$ and $v \leq C$ up to $T-\varepsilon$, so have found a neighborhood of $\left(u_{0}, v_{0}\right)$ in $L^{\infty}$ such that, if $(\hat{u}, \hat{v})$ has initial data in such neighborhood, then $\hat{u}$ becomes large at time $T-\varepsilon$ and $\hat{v} \leq C+1$ up to $T-\varepsilon$. The argument in the proof of Theorem 1.1 allows us to conclude that $\hat{u}$ blows up and $\hat{v}$ remains bounded, if we consider time $T-\varepsilon$ as the initial time.

Proof of Theorem 1.8 Under our assumptions, from Theorem 1.1, we know that the set of $\left(u_{0}, v_{0}\right)$ such that $u$ blows up and $v$ remains bounded is nonempty. From Corollary 1.4(i), we also know the set of initial data for $v$ blowing up and $u$ being bounded is nonempty. Moreover, Theorem 1.7 concludes that such sets are open. Clearly, the two open sets are disjoint. That is to say, there exist $\left(u_{0}, v_{0}\right)$ such that $u$ and $v$ blow up simultaneously at a finite time $T$. 
Next, we will get a simultaneously blow-up rate, if there are simultaneous blow-ups. Define

$$
M(t)=u(1, t)=\max _{[0,1]} u(\cdot, t), \quad N(t)=v(1, t)=\max _{[0,1]} v(\cdot, t), \quad \text { for each } t \in(0, T) .
$$

Set

$$
\begin{array}{ll}
\varphi_{M}(y, s)=\frac{1}{M(t)} u(a y+1, b s+t), & y \in[-1 / a, 0], s \in[-t / b, 0], \\
\psi_{N}(y, s)=\frac{1}{N(t)} v(c y+1, d s+t), & y \in[-1 / c, 0], s \in[-t / d, 0],
\end{array}
$$

where $a, b, c, d$ are positive functions of $t$ defined as follows:

$$
\begin{array}{ll}
a=\frac{M^{-\left(p_{1}-m\right)}}{N^{q_{1}}}, & b=\frac{M^{-\left(2 p_{1}-m-1\right)}}{N^{2 q_{1}}}, \\
c=\frac{N^{-\left(q_{2}-n\right)}}{M^{p_{2}}}, & d=\frac{N^{-\left(2 q_{2}-n-1\right)}}{M^{2 p_{2}}} .
\end{array}
$$

Then we have

$$
\begin{aligned}
& 0 \leq \varphi_{M}, \psi_{N} \leq 1, \\
& \varphi_{M}(0,0)=\psi_{N}(0,0)=1, \\
& \frac{\partial \varphi_{M}}{\partial s}=\frac{b}{M} \cdot u_{t} \geq 0, \quad \frac{\partial \psi_{N}}{\partial s} \geq 0 .
\end{aligned}
$$

Moreover, $\left(\varphi_{M}, \psi_{N}\right)$ satisfies the following problem:

$$
\left\{\begin{array}{l}
\left(\varphi_{M}\right)_{s}=\left(\varphi_{M}^{m}\right)_{y y}+\lambda_{1} k_{M}\left(\varphi_{M}\right)^{\alpha} \\
\left(\psi_{N}\right)_{s}=\left(\psi_{N}^{n}\right)_{y y}+\lambda_{2} k_{N}\left(\psi_{N}\right)^{\beta} \\
\left(\varphi_{M}^{m}\right)_{y}(0, s)=\varphi_{M}^{p_{1}} \psi_{N}^{q_{1}}(0, s) \\
\left(\psi_{N}^{n}\right)_{y}(0, s)=\varphi_{M}^{p_{2}} \psi_{N}^{q_{2}}(0, s)
\end{array}\right.
$$

where

$$
k_{M}=\frac{M^{-\left(2 p_{1}-m-\alpha\right)}}{N^{2 q_{1}}}, \quad k_{N}=\frac{N^{-\left(2 q_{2}-n-\beta\right)}}{M^{2 p_{2}}} .
$$

Obviously, $a, b, c, d, k_{M}, k_{N} \rightarrow 0$ as $t \rightarrow T$.

We claim that there exist positive constants $c$ and $C$, such that

$$
c \leq\left(\varphi_{M}\right)_{s}(0,0) \leq C, \quad c \leq\left(\psi_{N}\right)_{s}(0,0) \leq C
$$

for every small $a$.

Here, we only consider the estimate of $\left(\varphi_{M}\right)_{s}(0,0)$; that of $\left(\psi_{N}\right)_{s}(0,0)$ can be proven in a similar way. Given $\left\{\varphi_{M_{j}}\right\}$, there is a continuous function $\varphi$ and a subsequence, which we denote again by $\left\{\varphi_{M_{j}}\right\}$, such that $\varphi_{M_{j}} \rightarrow \varphi$ as $M \rightarrow \infty$. Therefore, there exists a neighborhood of $(0,0), U$, such that $\varphi>1 / 2$ in $U$. Since we have uniform convergence in $\bar{U}$ 
(we can assume that $\bar{U}$ is compact), for $j$ large enough, we have $1 / 4 \leq \varphi_{M_{j}} \leq 1 \mathrm{in} \bar{U}$. Thus the functions $\varphi_{M_{j}}$ are solutions of uniformly parabolic equations in $\bar{U}$, using the Schauder estimates

$$
\left\|\varphi_{M_{j}}\right\|_{C^{2+\alpha, 1+\alpha / 2}} \leq C, \quad \text { in } \bar{U}
$$

the upper bound in (3.1) follows immediately. To obtain the lower estimate, assume for finding a contradiction that there exists a sequence $\left\{\varphi_{M_{j}}, \psi_{N_{j}}\right\}$ such that $\left(\varphi_{M_{j}}\right)_{s}(0,0) \rightarrow 0$. Since $\varphi_{M_{j}} \rightarrow \varphi, \psi_{N_{j}} \rightarrow \psi, 0<\varphi, \psi \leq 1, \varphi(0,0)=\psi(0,0)=1, \frac{\partial \varphi}{\partial s}, \frac{\partial \psi}{\partial s} \geq 0$, and $\varphi$ satisfies

$$
\left\{\begin{array}{l}
\varphi_{s}=\left(\varphi^{m}\right)_{y y} \\
\left(\varphi^{m}\right)_{y}(0, s)=\varphi^{p_{1}}(0, s) \psi^{p_{2}}(0, s)
\end{array}\right.
$$

$w=\varphi_{s}$ satisfies

$$
\left\{\begin{array}{l}
w_{s}=\left(m \varphi^{m-1} w\right)_{y y} \\
\left(m \varphi^{m-1} w\right)_{y}(0, s)=p_{1} \varphi^{p_{1}-1} w(0, s) \psi^{p_{2}}(0, s)+p_{2} \varphi^{p_{1}} \psi^{p_{2}-1} \psi_{s}(0, s) \geq 0 .
\end{array}\right.
$$

On the other hand, $\left(\varphi_{M_{j}}\right)_{s}(0,0) \rightarrow \varphi_{s}(0,0)$, so $w(0,0)=\varphi_{s}(0,0)=0$, since $u_{t} \geq 0, w=\varphi_{s} \geq$ 0 , hence $w$ has a minimum at $(0,0)$, and by Hopf's lemma, $w \equiv 0$, which implies $\varphi$ does not depend on $s$. Thus $\varphi=\varphi(y)$ satisfies

$$
\left\{\begin{array}{l}
0=\left(\varphi^{m}\right)_{y y} \\
\left(\varphi^{m}\right)_{y}(0)=\varphi^{p_{1}}(0) \psi^{p_{2}}(0)=1
\end{array}\right.
$$

so $\left(\varphi^{m}\right)(y) \geq 1+y$, which contradicts the fact that $0 \leq \varphi \leq 1$; thus (3.1) holds.

Rewriting the estimate in terms of $M$ and $N$, we have

$$
c \leq M^{m-2 p_{1}} M^{\prime} / N^{2 q_{1}} \leq C, \quad c \leq N^{n-2 q_{2}} N^{\prime} / M^{2 p_{2}} \leq C,
$$

and hence

$$
\begin{aligned}
& c \leq \frac{M^{m-2 p_{1}+2 p_{2}} M^{\prime}}{N^{n-2 q_{2}+2 q_{1}} N^{\prime}} \leq C, \\
& c N^{n-2 q_{2}+2 q_{1}} N^{\prime} \leq M^{m-2 p_{1}+2 p_{2}} M^{\prime} \leq C N^{n-2 q_{2}+2 q_{1}} N^{\prime} .
\end{aligned}
$$

Integrating the above inequality on $(t, T)$ we obtain

$$
c \leq N^{\prime} N^{\frac{\left(n-2 q_{2}+1\right)\left(m-2 p_{1}+1\right)-4 p_{2} q_{1}}{m-2 p_{1}+2 p_{2}+1}} \leq C .
$$

Integrating on $(t, T)$, the estimate of $v$ follows. Through a similar computation the estimate of $u$ can be given.

\section{Non-simultaneous blow-ups always happen}

In this section, we will show that under certain conditions non-simultaneous blow-ups always occur, and we will prove Theorems 1.9 and 1.10 . 
Proof of Theorem 1.9 Assume that there is an initial data $\left(u_{0}, v_{0}\right)$, such that $u$ and $v$ both blow up at time $T$. Following the steps of the proof of Theorem 1.8, we get (3.2). Thus

$$
M^{m-2 p_{1}} M^{\prime} \geq c N^{2 q_{1}}, \quad N^{n-2 q_{2}} N^{\prime} \leq C M^{2 p_{2}} .
$$

First, we assume that $2 q_{1}-2 q_{2}+n+1>0$, after a straightforward computation we obtain

$$
C M(t)^{2 p_{2}-2 p_{1}+m+1}+C \geq c N(t)^{2 q_{1}-2 q_{2}+n+1}-c .
$$

As $2 p_{2}-2 p_{1}+m+1<0$, and $2 q_{1}-2 q_{2}+n+1>0$, we obtain a contradiction with the assumption of simultaneous blow-ups.

If $2 q_{1}-2 q_{2}+n+1=0$, we have

$$
C M(t)^{2 p_{2}-2 p_{1}+m+1}+C \geq c \ln N(t)-c
$$

also a contradiction.

Proof of Theorem 1.10 It is well known that under the assumptions, $v$ cannot blow up without the help of $u$. So the blow-up time of $v$ cannot be larger than that of $u$. $u$ blows up at finite time $T$, by considering the solution at time $T-\varepsilon$ as initial data, we may assume that the blow-up time is as small as desired. Following the same steps of the proof of Theorem 1.1, the conclusion follows.

\section{Competing interests}

The author declares that they have no competing interests.

Received: 11 July 2015 Accepted: 28 September 2015 Published online: 15 October 2015

\section{References}

1. Bebernes, J, Eberly, D: Mathematical Problem from Combustion Theory. Appl. Math. Sci., vol. 83. Springer, Berlin (1989)

2. Lieberman, GM: Second Parabolic Differential Equations. World Scientific, Singapore (1996)

3. Song, XF, Zheng, SN: Blow-up analysis for a quasilinear parabolic system with multi-coupled nonlinearities. J. Math. Anal. Appl. 281, 739-756 (2003)

4. Brändle, C, Quirós, F, Rossi, JD: Non-simultaneous blow-up for a quasilinear parabolic system with reaction at the boundary. Commun. Pure Appl. Anal. 4(3), 523-536 (2005)

5. Brändle, C, Quirós, F, Rossi, JD: The role of non-linear diffusion in non-simultaneous blow-up. J. Math. Anal. Appl. 308, 92-104 (2005)

6. Pinasco, JP, Rossi, JD: Simultaneous versus non-simultaneous blow-up. N.Z. J. Math. 29, 55-59 (2000)

7. Quirós, F, Rossi, JD: Non-simultaneous blow-up in a semilinear parabolic system. Z. Angew. Math. Phys. 52, $342-346$ (2001)

8. Quirós, F, Rossi, JD: Non-simultaneous blow-up in a nonlinear parabolic system. Adv. Nonlinear Stud. 3, 397-418 (2003)

9. Rossi, JD, Souplet, P: Coexistence of simultaneous and non-simultaneous blow-up in a semilinear parabolic system. Differ. Integral Equ. 18, 405-418 (2005)

10. Zheng, SN, Qiao, L: Non-simultaneous blow-up in a reaction-diffusion system. Appl. Comput. Math. 180, 309-317 (2006)

11. Zheng, SN, Liu, BC, Li, FJ: Non-simultaneous blow-up for a multi-coupled reaction-diffusion system. Nonlinear Anal. 64, 1189-1202 (2006)

12. Zheng, SN, Liu, BC, Li, FJ: Simultaneous and non-simultaneous blow-up for a cross-coupled parabolic system. J. Math. Anal. Appl. 326, 414-431 (2007)

13. Liu, BC, Li, FJ: Non-simultaneous blow-up and blow-up rates for reaction-diffusion equations. Nonlinear Anal. 13, 764-778 (2012)

14. Ling, ZQ, Wang, ZJ, Zhang, GQ: The simultaneous and non-simultaneous blow-up criteria for a diffusion system. Acta Math. Sci. 33(1), 139-149 (2013)

15. Jiang, ZX, Zheng, SN, Song, XF: Blow-up analysis for a nonlinear diffusion equation with nonlinear boundary conditions. Appl. Math. Lett. 17, 193-199 (2004)

16. Pablo, AD, Quirós, F, Rossi, JD: Asymptotic simplification for a reaction-diffusion problem with a nonlinear boundary condition. IMA J. Appl. Math. 67, 69-98 (2002) 
17. Song, $X F$, Zheng, SN: Blow-up and blow-up rate for a reaction-diffusion model with multiple nonlinearities. Nonlinear Anal. 54, 279-289 (2003)

18. Jiang, ZX, Zheng, SN: Blow-up rate for a nonlinear diffusion equation with absorption and nonlinear boundary flux. Adv. Math. 33, 615-620 (2004)

19. Friedman, A: Partial Differential Equations of Parabolic Type. Prentice-Hall, Englewood Cliffs (1964)

Submit your manuscript to a SpringerOpen ${ }^{\odot}$ journal and benefit from:

- Convenient online submission

- Rigorous peer review

- Immediate publication on acceptance

- Open access: articles freely available online

- High visibility within the field

- Retaining the copyright to your article

Submit your next manuscript at $\boldsymbol{s p r i n g e r o p e n . c o m ~}$ 